\title{
Tuberculoid Leprosy with External Jugular Vein Thrombosis: A Case Report and Literature Review
}

\author{
Ali Ibrahim Rahil ${ }^{1}$, Ahmed Osman ${ }^{1}$, Mohamed Magdi ${ }^{1}$, Sara Kanbour ${ }^{2}$, Ahmed Emad Mahfouz ${ }^{1}$ \\ ${ }^{1}$ Hamad General Hospital, Hamad Medical Corporation, Doha, Qatar \\ ${ }^{2}$ Weill Cornell Medical College, Doha, Qatar
}

Received:30/09/2019

Accepted: 26/11/2019

Published: 20/01/2020

\begin{abstract}
How to cite this article: Rahil AI, Osmed A, Magdi M, Kanbour S, Mahfouz AE. Tubercoloid leprosy with external jugular vein thrombosis: a case report and
\end{abstract} literature review. EJCRIM 2020;7: doi:10.12890/2020_001302.

Conflicts of Interests: The Authors declare that there are no competing interest

Acknowledgements: We would like to thank Editage (www.editage.com) for English language editing.

This article is licensed under a Commons Attribution Non-Commercial 4.0 License

\section{ABSTRACT}

Thrombotic disease represents a rare manifestation of leprosy. In this study, we report the case of an external jugular vein thrombosis associated with tuberculoid leprosy in a 23-year-old male patient. The patient presented with a 3-month history of painful cord-like swelling on the left side of the neck and a nearly 3-week history of skin lesions on the left cheek and right leg. Physical examination revealed cord-like, tender swelling on the left lateral aspect of the neck overlying the sternocleidomastoid muscle, and a hypopigmented, hypoaesthetic $6 \times 7 \mathrm{~cm}$ lesion with an irregular margin on the left cheek. A Doppler ultrasound examination of the jugular vein showed thrombosis of the left external jugular vein. Three-dimensional reconstruction of the computed tomography scan showed the enlarged and enhanced left external jugular vein, as well as 1 of its tributaries, and the thickened skin patch. A skin punch biopsy from the left cheek lesion revealed granulomatous inflammation with occasional peri-adnexal granulomas, consistent with the clinical impression of tuberculoid leprosy. A diagnosis of leprosy with external jugular vein thrombosis was established. Anticoagulation therapy was initiated, and the patient was referred to an infectious disease clinic for treatment with anti-leprosy medications.

\section{LEARNING POINTS}

- Thickened cord-like neck swelling in leprosy can be vein thrombosis rather than a thickened nerve.

- Leprosy should be considered if a skin lesion is associated with thrombosis.

- $\quad$ The common causes of upper extremity DVT.

\section{KEYWORDS}

Tuberculoid leprosy, jugular vein thrombosis, thickened nerve

\section{INTRODUCTION}

Upper extremity deep vein thrombosis remains an important condition, with pulmonary embolism complicating approximately one-third of all cases. The most common causes of upper extremity thrombosis are central venous catheterization, infections, intravenous drug use, trauma, abdominal surgery, compression due to a tumour or the use of a collar and cuff for humeral fracture stabilization, vessel abnormalities, such as saccular aneurysms of the external jugular vein and Lemierre's syndrome. There are several cases in the literature demonstrating an association between venous thrombosis and thalidomide used in the treatment of leprosy, particularly when used with steroids ${ }^{[1-6]}$; however, there has only been 1 case in the literature reporting an association between tuberculoid leprosy and external jugular vein thrombosis ${ }^{[7]}$. Therefore, we herein present the first case of tuberculoid leprosy associated with external jugular vein thrombosis in Qatar. 


\section{CASE DESCRIPTION}

This study reports the case of a previously healthy 23-year-old Nepalese man who presented with a 3-month history of painful cord-like swelling on the left side of his neck, a 2-month history of right middle finger swelling, and a 20-day history of skin lesions on the left cheek and right leg. The patient did not report a history of fever, trauma, intravenous drug use, surgery, neck instrumentation or weight loss, and had no personal or family history of venous or arterial thrombosis.

Physical examination revealed the following: a hypopigmented, hypoaesthetic $6 \times 7 \mathrm{~cm}$ lesion with an irregular margin on the left cheek; 2 tender cord-like, non-compressible swellings overlying the left sternocleidomastoid muscle (Fig. 1); swelling of the right middle finger associated with an electric shock-like sensation upon palpation; and a hypopigmented, hypoaesthetic skin lesion on the dorsum of the right leg.

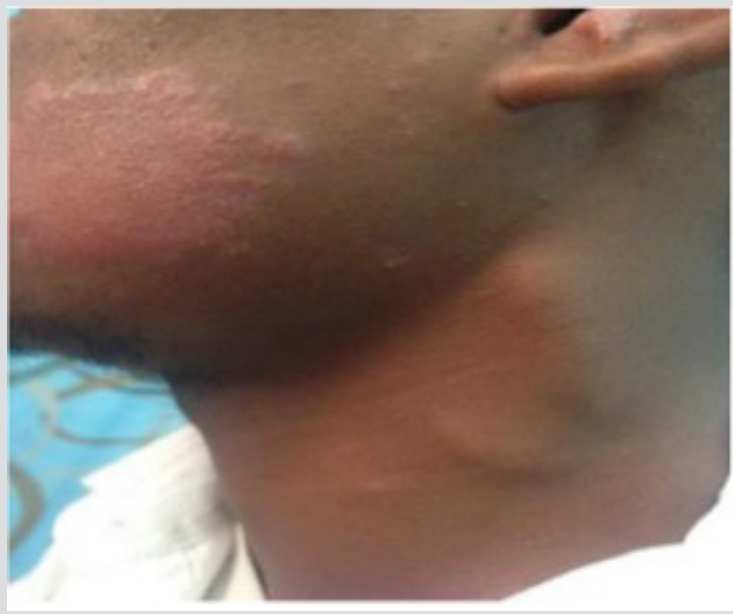

Figure 1. Hypopigmented patch on the left cheek and a cord-like swelling on the neck.

Furthermore, the pupils were of equal size and reactive to light, no motor deficits or sensory disturbances were observed (apart from the above-mentioned), and there were no palpable nerves. All other aspects of the systemic examination were unremarkable.

A Doppler ultrasound examination of the jugular vein showed thrombosis of the left external jugular vein, although the internal jugular veins on both sides showed normal flow. Furthermore, computed tomography scans of the head and neck were suggestive of left external jugular vein thrombosis (Figs. $2 \mathrm{~A}$ and $2 \mathrm{~B}$ ), whereas computed tomography scans of the chest, abdomen and pelvis were unremarkable.
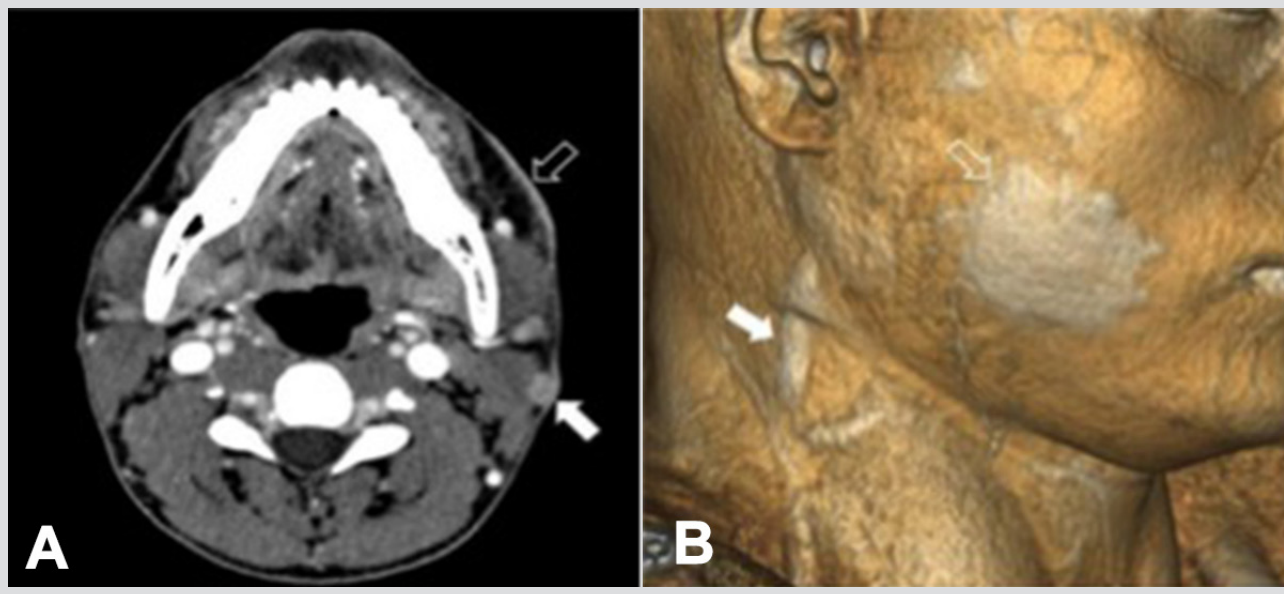

Figure 2. (A) Transverse contrast-enhanced computed tomography (CT) scan showing enlargement and occlusion of the left external jugular vein (solid arrow) and focal skin thickening of the left cheek (hollow arrow). Enhancement of the lumen of the occluded left external jugular vein excludes the possibility of $a$ bland thrombus and is indicative of lepromatous phlebitis. (B) Threedimensional reconstruction of the CT scan shows the enlarged and enhanced left external jugular vein, as well as one of its tributaries (filled arrow) and the thickened skin patches of the left cheek (unfilled arrow). 
Laboratory findings revealed the following: white blood cell count of $7.3 \times 10^{9} /$; haemoglobin concentration of $16.1 \mathrm{~g} / \mathrm{dl}$ (haematocrit: 48.4\%); platelet concentration of $290 \times 10^{9} / \mathrm{l}$; D-dimer concentration of $0.32 \mathrm{mg} / \mathrm{l}$ (normal concentration, <0.5 mg/l); prothrombin time of $10 \mathrm{sec}$ (normal range, 7.8-10 sec); activated partial thromboplastin time of $28 \mathrm{sec}$ (normal range, 25.3-33.8 sec); international normalized ratio of 1.1; protein C content of 93\% (normal range, 70-140\%); protein S content of 105\% (normal range, 60-130\%); antithrombin activity of $85 \%$ (normal range, 71- 16\%); anticardiolipin antibody levels of <9.3 MPL (normal levels, IgG <15 MPL and IgM <12.5 MPL); and were negative for antinuclear antibodies.

A skin punch biopsy from the left cheek lesion revealed granulomatous inflammation with occasional peri-adnexal granulomas, consistent with the clinical impression of tuberculoid leprosy. However, biopsy cultures were negative for leprosy bacilli (Fig. 3 and 4).

The patient was diagnosed with leprosy associated with external jugular vein thrombosis. Anticoagulation therapy was initiated with enoxaparin $1 \mathrm{mg} / \mathrm{kg}$ twice daily subcutaneously and warfarin $5 \mathrm{mg} /$ day, adjusted to achieve a target INR of 2-3, for a total of 3-6 months, according to the clinical response of the leprosy lesions to antibacterial therapy.

The patient was then referred to an infectious disease clinic for the commencement of leprosy treatment with dapsone 100 mg daily and rifampicin $600 \mathrm{mg} /$ day for 12 months. Unfortunately, the patient was lost to follow-up.

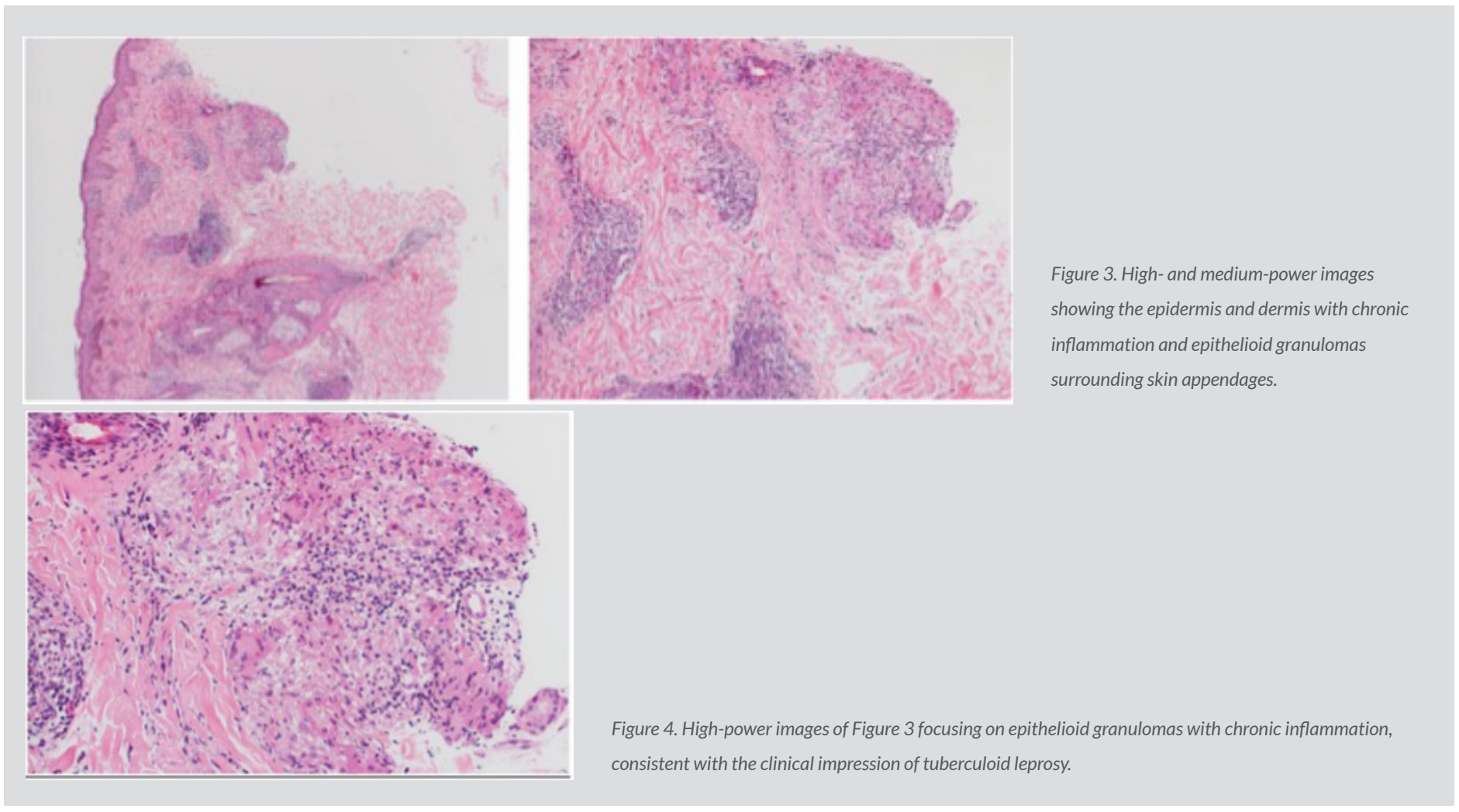

\section{DISCUSSION}

Leprosy is rare, and this is the first case of leprosy associated with external jugular vein thrombosis to be reported in Qatar. Leprosy is a disease that typically affects the skin and nerves. Vascular involvement in patients with leprosy is extremely rare, and is likely to occur secondary to involvement of the vasa nervorum blood vessels ${ }^{[8]}$. Intimal cell hyperplasia and vascular occlusion associated with lepromatous exudates were also observed in a small group study ${ }^{[9]}$.

In a previous study that assessed venous system involvement in leprosy patients using venography and vein histological analyses, a perfect correlation between the histological and venography findings was found in $40 \%$ of patients with paucibacillary leprosy, and a good correlation was observed in $100 \%$ of the patients. In patients with multibacillary leprosy, a perfect correlation was found in $29.4 \%$ of the patients, a good correlation in $41.1 \%$ of the patients and no correlation in $29.4 \%$ of the patients ${ }^{[10]}$. A thorough literature search yielded only a single prior documented case of tuberculoid leprosy associated with external jugular vein thrombosis, which occurred with severe disseminated disease ${ }^{[11]}$. 
Virchow's triad describes the factors contributing to venous thrombosis, which include hypercoagulability, stasis and endothelial injury ${ }^{[12]}$. Regarding hypercoagulability status, the patient did not report a personal or familial history of coagulation disorders. The diagnostic workup included evaluation of protein C and protein S deficiencies, as well as antithrombin III deficiency. Anticardiolipin antibodies were used to determine possible antiphospholipid syndrome. The laboratory values were within normal limits.

The patient did not have a history of recent surgery, prolonged immobilization, trauma to the area, neck instrumentation or a malignancy that would increase blood stasis or endothelial injury.

This study demonstrated that leprosy should be considered in patients who present with skin lesions in association with thrombotic features. This study reports the first case of leprosy associated with external jugular vein thrombosis in Qatar. Therefore, venous thrombosis should be considered an important potential clinical manifestation of leprosy.

\section{REFERENCES}

1. Vetrichevvel TP, Pise GA, Thappa DM. A case report of venous thrombosis in a leprosy patient treated with corticosteroid and thalidomide. Lepr Rev 2008;79:193-195.

2. Medeiros S, Fernandes C, Martins N, Machado J, Kutzner H, Afonso A, et al. Hansen's disease in an HIV patient complicated by deep vein thrombosis: a rare complication of thalidomide therapy. Eur J Dermatol 2009;19:272-273.

3. Thangaraju P, Giri VC, Aravindan U, Sajitha V, Showkath Ali MK. Ileofemoral deep vein thrombosis (DVT) in steroid treated lepra type 2 reaction patient. Indian J Lepr 2015;87:165-168.

4. Hebe Petiti-Martin G, Villar-Buill M, de la Hera I, Fuertes L, Burgués-Calderón M, Rivera-Díaz R, et al. Deep vein thrombosis in a patient with lepromatous leprosy receiving thalidomide to treat leprosy reaction. Actas Dermosifiliogr 2013;104:67-70.

5. Yamaguchi S, Yamamoto Y, Hosokawa A, Hagiwara K, Uezato H, Takahashi K. Deep venous thrombosis and pulmonary embolism secondary to co-administration of thalidomide and oral corticosteroid in a patient with leprosy. J Dermatol 2012;39:711-714.

6. Sharma NL, Sharma V, Shanker V, Mahajan VK, Sarin S. Deep vein thrombosis: a rare complication of thalidomide therapy in recurrent erythema nodosum leprosum. Int J Lepr Other Mycobact Dis 2004:72:483-485.

7. Zarza J, Centurión ME, Armoa A, Calvo M. [External jugular vein thrombosis associated with Hansen's disease.] Rev Clin Esp 2011;211:215-216.

8. Thakur S, Negi GS, Madabhavi I, Revannasiddaiah S. Lepromatous phlebitis of the left external jugular vein. BMJ Case Rep 2012;2012:bcr0120125473.

9. Mukherjee A, Girdhar BK, Malviya GN, Ramu G, Desikan KV. Involvement of subcutaneous veins in lepromatous leprosy. Int J Lepr Other Mycobact Dis 1983;51:1-6.

10. Bansal R, Kaur S, Kumar B, Sharma VK, Katariya S, Chakravarti RN, et al. Venous involvement in leprosy: a venographic and histopathological correlation. Int J Lepr Other Mycobact Dis 1987;55:499-506.

11. Thompson AM, Lynn AA, Robson K, Joyce MP, Fivenson DP, Scollard D. Lepromatous phlebitis of the external jugular vein. J Am Acad Dermatol 2003;49:1180-1182.

12. Malone PC, Agutter PS. Deep venous thrombosis: the valve cusp hypoxia thesis and its incompatibility with modern orthodoxy. Med Hypotheses 2016;86:60-66. 